\title{
An assessment framework for classifying determinants of household water consumption and their priorities for research and practice
}

\author{
A. Cominola ${ }^{\mathrm{a}, \mathrm{b}}$, L. Preiss ${ }^{\mathrm{c}}, \underline{\text { M. Thyer }}{ }^{\mathrm{c}, *}$, H. R. Maier ${ }^{\mathrm{c}}$, P. Prevos ${ }^{\mathrm{d}}$, R. Stewart ${ }^{\mathrm{e}, \mathrm{f}}$ and A. Castelletti ${ }^{\mathrm{g}}$ \\ ${ }^{a}$ Chair of Smart Water Networks, Technische Universität Berlin, Berlin, Germany \\ ${ }^{b}$ Einstein Center Digital Future, Berlin, Germany \\ ${ }^{c}$ School of Civil, Environmental and Mining Engineering, University of Adelaide \\ ${ }^{d}$ Victoria University, Melbourne, Australia \\ ${ }^{e}$ School of Engineering and Built Environment, Griffith University, Gold Coast, Australia \\ ${ }^{f}$ Cities Research Institute, Griffith University, Gold Coast, Australia \\ ${ }^{g}$ Department of Electronics, Information, and Bioengineering, Politecnico di Milano, Milan, Italy \\ Email: mark.thyer@adelaide.edu.au
}

\begin{abstract}
Achieving a thorough understanding of the determinants of household water consumption is crucial to support demand management strategies. Yet, existing research on household water consumption determinants is often limited to specific case studies, with findings that are difficult to generalize and not conclusive. Here, we contribute a framework for review, classification, and analysis of the literature on the determinants of household water consumption. Firstly, we identify a comprehensive set of 48 relevant publications, based on a systematic paper search. The framework firstly classifies household determinants into observable (physically seen/measured aspects of the house), latent (relates to the way occupants think/act/feel) and external (external to house and influence at regional level). Secondly, we undertake a trade-off analysis of different criteria that account for the representation of a potential water consumption determinant in the literature, its impact across heterogeneous case studies, and the effort required to collect information on it. The results of our trade-off analysis show that distinct groups of determinants exist, allowing for the formulation of four recommendation categories. These provide guidance for practitioners on which determinants to consider in practice and for researchers to prioritize in future research (Figure 1).
\end{abstract}

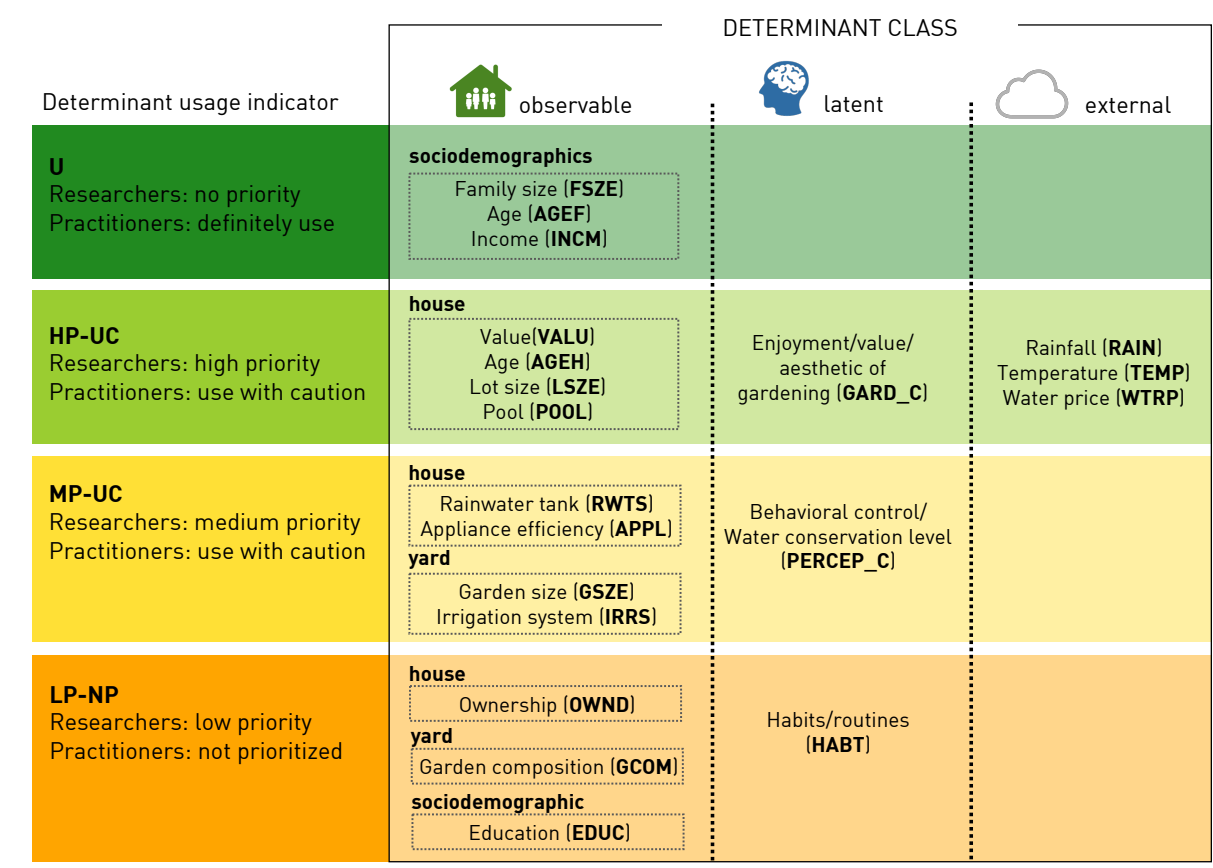

Figure 1. Recommendations for practitioners and researchers for individual determinants.

Keywords: household water consumption, water consumption determinants, water demand management, smart meter, review 
Cominola, An assessment framework for classifying determinants of household water consumption and their priorities for research and practice

\section{INTRODUCTION}

As urbanization is increasing globally, water demand-side management strategies are emerging as key interventions to manage the current and future urban metabolism and realize the potential of water conservation in cities (Turner et al. 2010). The domestic sector uses one of the largest portions of water in cities (Arbon et al. 2014). Therefore, achieving a thorough understanding of how, when, and how much water is used in households is of the upmost importance for water authorities and policy makers alike to design effective demand-side management strategies and inform future urban planning (Jorgensen et al. 2009). Knowledge of the behaviour surrounding water demand is vital to positively influence water conservation (Russell and Fielding 2010) and to implement effective and enforceable water demand management strategies. Additionally, better knowledge and improved predictions of water demands would also allow water authorities to better size new water storage, distribution, and treatment infrastructure, as well as future upgrades of current systems (Donkor et al. 2014).

Recent literature has shown that there is a large number of climate and socio-demographic variables, attitudes, beliefs, and other factors that can vary between different households and can potentially influence water consumption. These factors, called determinants, are all potentially able to influence household water use in different ways and to varying degrees, depending on the context. In addition, the collection of data on each determinant is just as varied as the determinant itself. Some, such as average temperature, are readily accessible on a regional scale and can be obtained and analysed with ease (Bernardo et al. 2015). Others, such as people's perception of behavioral control, are difficult to capture. They represent subjective and stated information, rather than measurable observations, and often require detailed surveys with multiple questions, followed by lengthy and time-consuming analysis, to determine whether any information can be inferred from the survey data (Fielding et al. 2012).

Despite the advances described above, most of the state-of-the-art determinant analyses to date are often limited to a few case studies or to specific determinants, and the existing findings are difficult to generalize and not conclusive. To address this gap, we developed an assessment framework for review, classification, and analysis of the literature on household water consumption determinants. The aim is to determine the relevance of potential determinants for water consumption modelling and management, their proven impact on heterogeneous case studies, and the cost of labour and/or of equipment required to collect information about a determinant. This leads to a classification system of household water use determinants and recommendations for researchers and practitioners that can be used to inform future research and applications. This conference paper provides a summary of the key points of a journal paper (Cominola et al. 2021) - refer to this paper for further details.

\section{METHODOLOGY}

\subsection{Paper search and exclusion criteria}

To gain an understanding of the current state of research on the determinants of household water consumption, we systematically searched for relevant peer-reviewed journal papers. We began this search by using the following combinations of keywords:

$$
\begin{gathered}
\text { House* AND water } \\
\text { AND (use OR demand OR Consumption) } \\
\text { AND (psychology* OR influenc* OR driver* OR factor* or cause*) }
\end{gathered}
$$

in the subject/title/abstract of papers published in the last 40 years and stored in the Elsevier "Engineering Village" databases (Elsevier 2020). Using these keywords and the "limit to" feature returned a list of 4326 papers, from an initial list of over 8200 . Second, we screened the title and abstract of each paper for relevance within the scope of our study. This step returned a total of 238 papers referred to as the general water consumption set. Some of these general water consumption-related papers are commonly cited within the literature found in the search. We reference them in the motivation of this study or discussion surrounding the results, but they were not analysed as part of the water consumption determinant assessment framework.

Finally, we reviewed all the general water consumption-related papers to determine if they actually analysed determinants against water use results, which is the main requirement for a paper to be included in our systematic review. Additionally, we excluded papers that were non-residential settings, theoretical models, focused on single end-use or classified into indoor/outdoor use only, or papers that were water use related but did not focus on analysing determinants' relationships with water use (e.g., focused only on price elasticity), or papers that only focused on apartments. This resulted in a database of 48 papers used for systematic review 
Cominola, An assessment framework for classifying determinants of household water consumption and their priorities for research and practice

using the assessment framework. This set of papers is hereafter referred to as the set of framework analysis papers (see Cominola et al. 2021 for complete list).

\subsection{Water consumption determinant assessment framework}

\section{Determinant Classification}

We classify the water consumption determinants in three main categories: observable, latent, and external.

Observable determinants are defined as determinants that can be physically seen or measured. They can be easily and/or directly measured and include objective features related to the occupants of the household and their house (e.g., occupant age, household size, household income, number of toilets).

Latent determinants relate to the way the occupants of the household think, feel, or act. Typically, they cannot be directly measured and need to be inferred from surveys/direct questions, and can be subjective. Examples include attitude to water saving, individual habits, and beliefs.

External determinants are external to the house and might influence a suburb or groups of houses at a regional level. Examples might include weather variables, such as rainfall and/or temperature.

These three categories are used to facilitate the characterization and analysis of the determinants found in the literature, both in terms of ease of information gathering and impact on water consumption. For instance, observable determinants are generally easier to collect information on and, therefore, are expected to be more common in literature than latent determinants, which require more effort, as they are typically based on surveys of occupants. An overview of the determinant classification system is provided in Figure 2. As shown in the figure, we further separated each of the three categories above into sub-categories to group the individual determinants that were closely related into the same categories.

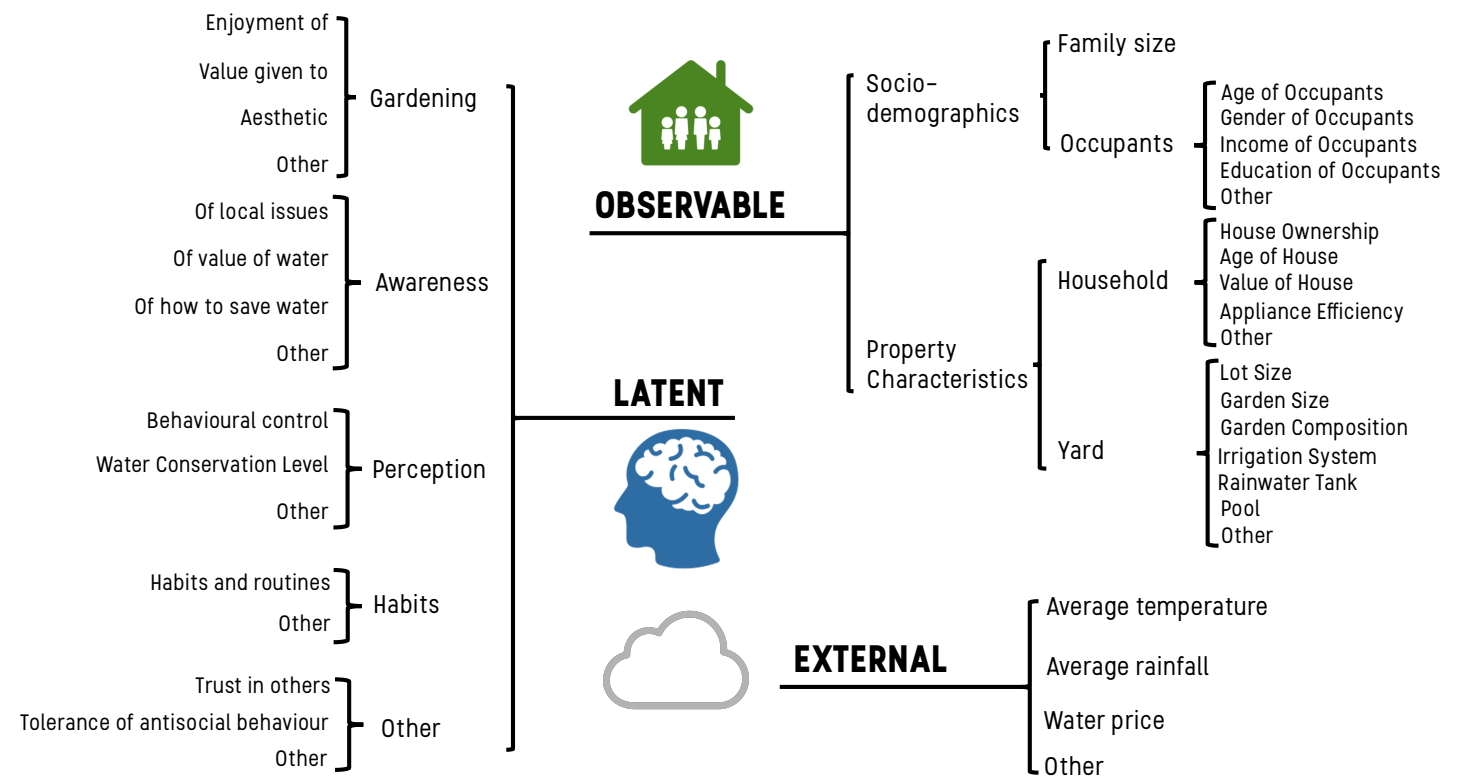

Figure 2. Overview of the determinant classification system

The observable determinants are broken down into two sub-categories. The first is socio-demographics, which includes Family Size (FSIZE), Age of Occupants (AGEF), Gender of Occupants (GNDR), Income of Occupants (INCM), and Education of Occupants (EDUC). The second is related to property characteristics (relating to the structure of the house itself), which includes House Ownership (OWND), Age of House (AGEH), Value of House (VALU), Appliance Efficiency (APPL), and multiple yard characteristics (relating to the yard and its irrigation). These latter include the Lot Size (LSIZE), Garden Size (GSIZE), Garden Composition (GCOMP), Irrigation System (IRRS), Rainwater tank (RWTS) and Pool (POOL).

Latent determinants are separated into the following sub-categories: The first is gardening (GARD_C), which relates to the 'enjoyment of value given to gardening' (ENJG/VALG) or 'appreciations of aesthetic aspects of gardening' (AESG). The second is awareness, (AWARE_C), which relates to awareness of either local issues, value of water or how to save water, etc. The third is perceptions (PERCP_C), which relates to how much 
Cominola, An assessment framework for classifying determinants of household water consumption and their priorities for research and practice

occupants perceive they have control over their behavior or their perceived water conservation (saving/wasting water). The fourth is habits (HABT), which relates to habits and routines of the occupants.

External determinants are sub-categorized depending on the variable of interest, i.e., average temperature (TEMP), average rainfall (RAIN), and water price (WTRP).

\section{Determinant analysis}

We defined three criteria to perform the determinant analysis: representation, importance, and effort.

Representation refers to how popular a determinant is in the reviewed literature on household water consumption. The representation $R_{i}$ of a determinant $i$ is defined as the relative frequency with which the determinant appeared in the set of framework analysis papers: $R_{i}=N_{i} / T$, where $N_{i}$ is the number of papers with determinant $i$ and $T$ is total number of framework analysis papers (i.e., 48).

Impact refers to whether or not a particular determinant actually influences the water use in a household. If the measurement and inclusion of a determinant was found to change the accuracy of a prediction or have some other effect on the household water demand, then the determinant was said to have impact, based on the following three possible categories of impact:

(i) Yes (Y). Impact found and analysed: determinant information was collected in the study, numerical analysis was undertaken (e.g., statistical analysis, regression) and the determinant was found to have an impact on demand/predictability by the authors of the paper. A blanket "Yes" rating was given to all determinants where impact was found, rather than assigning a low, medium, high impact rating because each paper used a different technique for assessing impact.

(ii) $\mathrm{No} / \mathrm{Low}(\mathrm{NL})$. Impact found and analysed: determinant information was collected in the study, numerical analysis was undertaken (e.g., statistical analysis, regression), but the determinant was found to have no or low impact on demand/predictability.

(iii) Collected, but not analysed (CNA). Determinant information was collected in the study, but no analysis was undertaken for reasons such as lack of sufficient data or not selecting the determinant as a focus.

The impact, $I_{i}$, for a given determinant $i$ is defined as $I_{i}=N_{i}^{Y e s} /\left(N_{i}^{Y e s}+N_{i}^{N o}\right)$, where $N_{i}^{Y e s}$ is the number of papers where the impact is "Yes" for determinant $i$, and $\left(N_{i}^{Y e s}+N_{i}^{N o}\right)$ is the total number of papers where the impact was "Yes" or "No" for the same determinant.

Effort refers to the cost of labour and/or of equipment required to collect information about a determinant. For the purposes of this paper, effort is divided into three categories, i.e., low, medium, and high. A low rating means that the information on the determinant is easily available for each house and can be obtained via a desktop study (e.g., census data), with no interaction with the households needed. Information with a medium rating requires some cost to collect, but no house visit is required (e.g., online/paper survey, telephone call, etc.) In contrast, the high effort rating corresponds to actually visiting the house and taking measurements and/or surveys. Each of the categories is quantified by a corresponding effort rating factor based on an estimate of the number of hours of labour required to collect information on a given determinant. This rating factor was then scaled such that the low rating is coupled with an effort rating factor equal to 1 , a medium effort with a rating factor of 10 , and a high effort factor with a rating factor of 100 .

The overall effort rating, $E_{i}$, for a given determinant, $i$, was determined as: $E_{i}=\sqrt[T_{i}]{1^{N_{L}^{i}} \times 10^{N_{M}^{i}} \times 100^{N_{H}^{i}}}$, where $N_{L}^{i}, N_{M}^{i}$ and $N_{H}^{i}$ are the number of studies reporting determinant $i$ with low (L), medium (M), or high (H) effort, respectively, and $T_{i}$ is the total number of papers considering that determinant. We used the geometric mean, rather than arithmetic mean, because the effort rating factor varies across two orders of magnitude.

\section{RESULTS}

\subsection{Trade-off analysis of representation, impact, and effort criteria}

Figure 3 shows the interaction between the representation, impact, and effort criteria. The distribution of blue and orange points in the figure demonstrates that there are different trade-offs among the three criteria. Each trade-off can have a different set of implications to derive recommendations for researchers and practitioners. We identified the three groups of determinants marked with (A), (B), and (C) to illustrate the different needs of research and practice. Group A is characterized by high impact, high representation, and low effort. Determinants in this group include household family size, occupants' age, and occupants' income. This group of well-studied determinants with proven impact might be particularly interesting for practitioners aiming to 
Cominola, An assessment framework for classifying determinants of household water consumption and their priorities for research and practice

gather knowledge on household water consumption with budget constraints. Group B includes information on the garden composition/size, appliance efficiency, and occupant gender. It is characterized by medium-to-high impact, but low representation, and a range of low to high efforts. While this group might not be very appealing for practitioners due to low representation, researchers might be interested in focusing on these determinants to validate or contrast the limited findings on these determinants that appear in the literature. Finally, Group $\mathrm{C}$ refers to determinants with low representation and low impact, which should be therefore handed carefully.

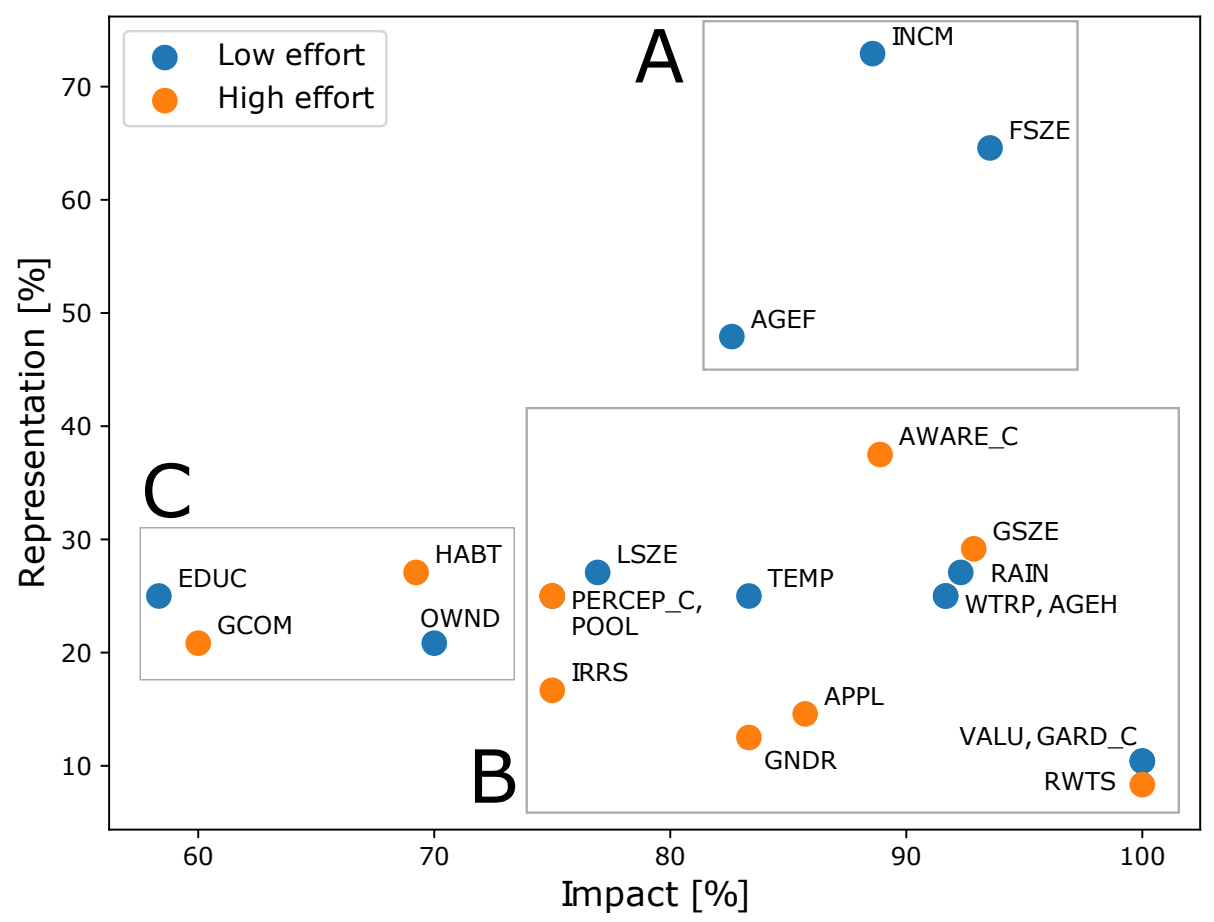

Figure 3. Impact (x-axis) vs Representation (y-axis) vs Effort (color) of each determinant. Each point refers to a specific determinant. The determinants classified as "High effort" are those with an overall effort value larger than 8.0, vice-versa for the "Low effort" determinants.

\subsection{Development of recommendation categories}

The trade-off analysis leads to the following four main recommendation categories (Table 1).

Use $(\boldsymbol{U})$. In this category, we include determinants characterized by high representation, high/very high impact, and low effort. We consider these determinants as determinants that practitioners can "definitely use" (U), as they have been extensively researched and have been shown to have an impact, while being affordable. For these reasons, higher levels of research priority should be devoted to less explored determinants. The determinants included in box (A) in Figure 3 belong to this group.

High Priority research - Use with Caution (HP-UC). In this category, we classify those determinants characterized by low representation, high/very high impact, and low effort. Given their promising, but not extensively proven, impact, and overall affordability, further research on these determinants should be highly prioritized (HP). We consider these determinants as determinants that practitioners can "use with caution" (UC), as they have not been extensively researched, but at the same time might have high impact at low-cost. The determinants included in box (B) in Figure 3 and classified as low effort (blue color) belong to this group.

Medium Priority research - Use with Caution (MP-UC). In this category, we include determinants characterized by generally low representation, high/very high impact, and high effort. Similarly to the previous category, we believe that practitioners can use these determinants "with caution" (UC), as they have not been extensively researched and require high effort for data collection, but at the same time might have high/very high impact. Given their promising, but not extensively proven, impact, and high cost, further research on these determinants should be prioritized with medium priority (MP). The determinants included in box (B) in Figure 3 and classified as high effort (orange color) belong to this group. 
Cominola, An assessment framework for classifying determinants of household water consumption and their priorities for research and practice

Low Priority research - Not Prioritized for use (LP-NP). In this category, we include determinants characterized by low representation, low impact, and mainly high effort. Given the limited knowledge on these determinants, likely low impact, and estimated high effort, research can be done with lower priority (LP), compared to other groups. We also suggest that these determinants are "not prioritized" (NP) for use by practitioners unless further research demonstrates that the effort required is worth the benefit. The determinants included in box (C) in Figure 3 belong to this group.

Table 1. Framework for trade-off analysis, based on representation, impact, and effort.

\begin{tabular}{|c|c|c|c|c|c|}
\hline $\begin{array}{c}\text { Determinant } \\
\text { usage indicator }\end{array}$ & Impact & Representation & Effort & $\begin{array}{l}\text { Recommendation } \\
\text { for researchers }\end{array}$ & $\begin{array}{l}\text { Recommendation } \\
\text { for practitioners }\end{array}$ \\
\hline $\mathbf{U}$ & (Very) High & High & Low & $\begin{array}{l}\text { No further research } \\
\text { to prioritize. }\end{array}$ & Definitely use. \\
\hline HP-UC & (Very) High & Low & Low & $\begin{array}{l}\text { High priority } \\
\text { research to } \\
\text { establish impact. }\end{array}$ & $\begin{array}{l}\text { Use with caution } \\
\text { until further } \\
\text { research. }\end{array}$ \\
\hline MP-UC & (Very) High & (Mainly) Low & High & $\begin{array}{l}\text { Medium priority } \\
\text { research to } \\
\text { establish impact } \\
\text { and/or reduce effort. }\end{array}$ & $\begin{array}{l}\text { Use with caution } \\
\text { until further } \\
\text { research. }\end{array}$ \\
\hline LP-NP & Low & Low & (Mainly) High & $\begin{array}{c}\text { Low priority } \\
\text { research to } \\
\text { establish impact } \\
\text { and/or reduce effort. }\end{array}$ & $\begin{array}{l}\text { Do not favour } \\
\text { adoption until } \\
\text { further research. } \\
\text { Not prioritized. }\end{array}$ \\
\hline
\end{tabular}

\subsection{Classification of Determinants into Recommendation categories}

Based on the trade-off analysis and the recommendation categories we associated each determinant (Figure 2) into one of four recommendation categories (Figure 1). Some relevant insights for researchers and practitioners emerge. First, only observable determinants are classified as "U". At present, there are some socio-demographic determinants (i.e., number of occupants, income level, and occupant age) that can be reliably used by practitioners in most cases to model household water consumption and can be easily and affordably retrieved.

Second, all external variables (i.e., average rainfall, temperature, and water price) are classified as HP-UC. Consequently, they have a proven impact, but have been used sporadically in connection with household water consumption (while they have been used more often at larger, urban scales), thus results might be case-specific and further research (high priority) is needed.

Third, a mix of observable and latent external variables deserve medium priority for research. These variables are either observable determinants, the collection of which requires significant effort and house visits/calls to occupants (e.g., to build an inventory of appliance efficiency or storing information on irrigation systems), or latent variables, the impact of which is still not proven due to low representation. The increasing availability of high resolution metering and behavioral studies fostered by smart metering development is likely to contribute more knowledge on these determinants and more complete guidelines for use by practitioners in the coming years (Arbon et al. 2014; Cominola et al. 2015; Stewart et al. 2018).

Finally, while there are some determinants classified as "do not prioritize for use and low-priority for research" (LP-NP), we would like to stress that this is not suggesting that no research should be done on them. Joint research that also includes other determinants with higher levels of priority could be beneficial to discover more on the determinants in this group and better understand whether practitioners should eventually include one/more of these determinants in their analysis.

\section{CONCLUSIONS}

In this paper, we contributed a comprehensive literature review and assessment framework to evaluate stateof-the-art research on the determinants of household water consumption. Starting from a search that returned over 8200 papers, we identified 48 papers that clearly identify whether a particular determinant can have an impact on household water demand. We then developed a classification system and assessment framework to analyse these 48 papers. First, we classified the potential determinants of household water consumption into three main categories, i.e., observable, latent, and external based on their nature and ease of information 
Cominola, An assessment framework for classifying determinants of household water consumption and their priorities for research and practice

retrieval. Second, we defined three quantitative criteria to analyse the influence of different determinants in relation to water consumption and quantified them for the determinants identified in the reviewed papers. These three indicators look at (i) how frequently a determinant appears in the literature (representation), (ii) whether a particular determinant has been observed to influence household water consumption (impact), and (iii) what the cost for labour and/or equipment required to collect information on a particular determinant is (effort).

Our trade-off analysis of representation, impact, and effort shows that there are some distinct groups of water consumption determinants. Each group has different implications for practitioners and researchers and our analysis provides valuable guidance for practitioners and researchers on which determinants to consider in a range of situations. The first group identified was observable determinants with high impact, high representation, and low effort (e.g., household family size, occupants' age, and income). These are recommended for use by practitioners as they been widely studied in the literature, have demonstrated impact, and can be obtained with low effort. The second and third groups were determinants with high impact, but low representation and with high or low effort. As these determinants had a limited number of studies (low representation) which correlated these determinants with water consumption at the household scale, it is recommended that practitioners "Use with caution". At the same time, these determinants had high impact, so they are recommended as priority for further research. "High" priority was given to determinants with low effort, which include information on the external determinants (e.g., climate variables and water price) and observable determinants (e.g., house value/age, lot size, pool), which are generally easily accessible and do not require ad hoc data gathering campaigns. "Medium" priority, was given to determinants with "high" effort. These include some other observable determinants (e.g., garden size) and most latent determinants that relate to subjective perceptions, awareness, habits, or opinions, which require a higher data gathering effort. Given the higher cost and more uncertain return, further analysis can be prioritized by researchers before use in practice.

\section{REFERENCES}

Arbon, N., Thyer, M., Hatton MacDonald, D., Beverley, K., Lambert, M., 2014. Understanding and predicting household water use for Adelaide. Goyder Institute for Water Research Technical Report Series 14, 15.

Bernardo, V., Fageda, X., Termes, M., 2015. Do droughts have long-term effects on water consumption? evidence from the urban area of barcelona. Applied Economics 47, 5131-5146.

Cominola, A., Giuliani, M., Piga, D., Castelletti, A., Rizzoli, A.E., 2015. Benefits and challenges of using smart meters for advancing residential water demand modeling and management: A review. Environmental Modelling \& Software 72, 198-214.

Cominola, A., Preiss, L., Thyer, M., Maier, H. M, Prevos, P. Stewart, R. Castelletti, A., 2021. The determinants of household water consumption: a review and assessment framework for research and practice, submitted to npj Clean Water.

Donkor, E.A., Mazzuchi, T.A., Soyer, R., Alan Roberson, J., 2014. Urban water demand forecasting: review of methods and models. Journal of Water Resources Planning and Management 140, 146-159.

Elsevier, 2020. Engineering Village. https://www.elsevier.com/solutions/engineering-village (Dec. 2020).

Fielding, K.S., Russell, S., Spinks, A., Mankad, A., 2012. Determinants of household water conservation: The role of demographic, infrastructure, behavior, and psychosocial variables. Water Resources Research 48.

Jorgensen, B., Graymore, M., O’Toole, K., 2009. Household water use behavior: An integrated model. Journal of environmental management 91, 227-236.

Russell, S., Fielding, K., 2010. Water demand management research: A psychological perspective. Water resources research 46 .

Stewart, R.A., Nguyen, K., Beal, C., Zhang, H., Sahin, O., Bertone, E., Vieira, A.S., Castelletti, A., Cominola, A., Giuliani, M., et al., 2018. Integrated intelligent water-energy metering systems and informatics: Visioning a digital multi-utility service provider. Environmental Modelling \& Software 105, 94-117.

Turner, A., Willetts, J., Fane, S., Giurco, D., Chong, J., Kazaglis, A., White, S., 2010. Guide to demand management and integrated resource planning (update on original 2008 guide) 